\title{
Online Center of Gravity Estimation in Automotive Vehicles using Multiple Models and Switching
}

\author{
Selim Solmaz \\ Hamilton Institute, NUI-Maynooth \\ Co. Kildare, Ireland \\ Email: selim.solmaz@nuim.ie \\ Telephone: +(353) 17086100 \\ Fax: +(353) 17086269
}

\author{
Mehmet Akar \\ Hamilton Institute, NUI-Maynooth \\ Co. Kildare, Ireland \\ Email: mehmet.akar@nuim.ie
}

\author{
Robert Shorten \\ Hamilton Institute, NUI-Maynooth \\ Co. Kildare, Ireland \\ Email: robert.shorten@nuim.ie
}

\begin{abstract}
In this paper we present a methodology based on multiple models and switching for realtime estimation of center of gravity (CG) position in automotive vehicles. The method utilizes simple linear vehicle models and assumes availability of standard stock automotive sensors. We demonstrate the efficacy of our technique with numerical simulations. We also give a simple application example for implementing the idea in automotive vehicles as a switch for rollover controller activation.
\end{abstract}

\section{INTRODUCTION}

Assessment of a road vehicle's handling behavior, performance characteristics and accident behavior is primarily correlated with the vehicle's center of gravity (CG) position as well as its inertial properties. Although automotive manufacturers often provide the measurement of these parameters, such information often pertain to an empty vehicle with known load distribution. Considering the fact that passenger and/or load distribution in road vehicles can vary significantly, and sometimes even dangerously, it is difficult to overlook the change in the CG position and its consequences. While the importance of this is known on the handling behavior, automotive manufacturers usually employ robust active road-handling control strategies to account for the unknown and changing CG position; they simply design for the worst case scenario. Another common approach in the case of Sport Utility Vehicles (SUVs) and pickup trucks is to intentionally design the vehicle heavier than usual by adding ballast in the undercarriage, which aims to lower the CG position while reducing the percent margin of the load variation and thus constraining the variation of the CG location. While such approaches are successful up to certain extent, they also come with obvious drawbacks; performance loss under normal driving conditions, requirement of a bigger engine, and efficiency loss due to added weight.

Analysis of a recent car accident data indicates that vehicles with a high center of gravity such as vans, trucks and SUVs are more prone to rollover accidents than others [1]. Also it is known that rollover accidents alone constitute only a small percentage of all car accidents, while they cause disproportionately high rates of fatalities [2]. According to recent statistics [1], rollover occurred in only $2.6 \%$ of all vehicle crashes during 2004 in the USA, while it was responsible for a massive $20.5 \%$ fatality rate, rendering it to be the most dangerous type of accident. Again according to the same data, light trucks (pickups, vans, SUVs) were involved in nearly $70 \%$ of all the rollover accidents, with SUVs alone responsible for almost $35 \%$ of this total. Considering the fact that composition of the current automotive fleet consists of nearly $36 \%$ light trucks, minivans and SUVs [3] along with the recent increase in the popularity of SUVs worldwide, makes the rollover an important safety problem. As CG height is the most prominent factor in un-tripped rollover occurrence, this problem can greatly benefit from real-time CG position estimation capabilities. Such estimators can be used as a warning system to the driver or can conveniently be integrated into active road handling or rollover prevention controllers thus improving the overall vehicle and passenger safety. In order to demonstrate how this can be implemented in a real vehicle, we give a simple application example, where CG estimation method is used as a switch for the rollover controller activation depending on the loading condition of the vehicle.

With this background in mind, and inspired by the Multiple Model Switching \& Tuning (MMST) methodology [4], we present in this paper a multiple model and switching estimation algorithm based on simple linearized models and employing only standard stock automotive sensors [5]. While simplified linear models such as the single track model (i.e., bicycle model) and the roll plane model can represent the real vehicle behavior in a limited range of maneuvers and speeds, it is possible to use a multitude of these models and switch between them in an intelligent way in real time, to track the vehicle behavior accurately over the complete operating conditions. Moreover, proper parametrization of these models gives way to the estimation of unknown and time-varying vehicle parameters through the selected models. Using the described multimodel approach in conjunction with linear roll plane models, one can estimate parameters such as the CG height and linear suspension parameters in relation to the rollover prevention problem, while through a similar implementation of multiple single track models one can estimate parameters relevant to lateral dynamics control, such as the longitudinal CG position and linear tire stiffnesses. One of the benefits of this realtime estimation method is the fact that the method is immune to the nonlinear dependance of unknown vehicle parameters in 
the models.

Recent publications related to automotive CG position measurement and estimation include that of Mango [6], where he described a method for accurately calculating the $\mathrm{CG}$ location based on portable wheel scales. His method requires external measurement equipment and is not intended for online measurement during regular driving conditions as it requires the vehicle to be stationary. In another recent article, Allen et al. [7] made a statistical analysis of vehicle inertial properties and $\mathrm{CG}$ positions as a function of weight, width, length and the height of the vehicle using the data for several existing stock cars. Although their analysis is useful in demonstrating the relationship between the several physical parameters of vehicles to their handling characteristics, their method can not be employed for realtime estimation purposes. As a last remark we note in the context of rollover prevention that all the methods suggested to date assume a known CG height [2], [3], [8], [9], [10], [11], [12], [13]. However as we have explained, it is particularly unrealistic to assume the $\mathrm{CG}$ height to be known, as this parameter can vary significantly with changing passenger and loading conditions especially in large passenger vehicles such as SUVs and there may be significant performance issues related to robust rollover controller designs.

\section{VEHICLE MODELLING}

In this section we present three different models for the lateral motion and roll plane dynamics of a car. We use linear models to simplify the implementation of the algorithm as well as to keep the required sensory information at a minimum level. Note that the selection of the models here is a trade off between complexity and sensitivity to different operating conditions. While linear models are valid only in a short horizon, switching between several of them eliminates the insensitivity of these simple models.

In this paper we assume the availability of lateral acceleration $a_{y}$, yaw rate $\dot{\psi}$, velocity $v_{x}$ and the steering angle $\delta$ measurements which are available as part of the standard sensor packs found in modern cars. Moreover we require measurement or an estimation of the vehicle roll angle. Spring displacement sensors commonly found in many stock SUVs can be employed for this purpose.

Notation and definitions of the model parameters and variables are given in Table I. We assume that vehicle mass $m$ is known, which can be estimated as part of the braking system, yet this is outside scope of this paper. Furthermore $C_{v}, C_{h}, l_{v}, k, c$ and $h$ are all assumed be unknown parameters of the vehicle and are estimated through the multiple model switching algorithm. We further assume that these parameters vary within certain closed intervals $C_{v} \in \mathscr{C}_{v}, C_{h} \in \mathscr{C}_{h}, l_{v} \in \mathscr{L}_{v}$, $c \in \mathscr{C}, k \in \mathscr{K}$ and $h \in \mathscr{H}$, and these intervals can be found via accurate numerical simulations as well as field tests.

In what follows we give three different dynamical equations of the motion of the car. For a through coverage of the derivations see [14], and [15]. Also note that, for simplicity, we assumed in the following relations that relative to the ground
TABLE I

Model Parameters AND Definitions

\begin{tabular}{cll}
\hline Parameter & Description & Unit \\
\hline$m$ & Vehicle mass & {$[\mathrm{kg}]$} \\
$g$ & Gravitational constant & {$\left[\mathrm{m} / \mathrm{s}^{2}\right]$} \\
$v_{x}$ & Vehicle longitudinal speed & {$[\mathrm{m} / \mathrm{s}]$} \\
$\delta$ & Steering angle & {$[\mathrm{rad}]$} \\
$J_{x x}$ & Roll moment of inertia at CG & {$\left[\mathrm{kg} \cdot \mathrm{m}^{2}\right]$} \\
$J_{z z}$ & Yaw moment of inertia at CG & {$\left[\mathrm{kg} \cdot \mathrm{m}^{2}\right]$} \\
$L$ & Axle separation & {$[\mathrm{m}]$} \\
$T$ & Track width & {$[\mathrm{m}]$} \\
$l_{v}$ & longitudinal CG position from the front axle & {$[\mathrm{m}]$} \\
$l_{h}$ & longitudinal CG position from the rear axle & {$[\mathrm{m}]$} \\
$h$ & CG height & {$[\mathrm{m}]$} \\
$c$ & suspension damping coefficient & {$\left[\mathrm{kg} \cdot \mathrm{m}^{2} / \mathrm{s}\right]$} \\
$k$ & suspension spring stiffness & {$\left[\mathrm{kg} \cdot \mathrm{m}^{2} / \mathrm{s}^{2}\right]$} \\
$C_{v}$ & front tire tire stiffness & {$[\mathrm{N} / \mathrm{rad}]$} \\
$C_{h}$ & rear tire tire stiffness & {$[\mathrm{N} / \mathrm{rad}]$} \\
$\beta$ & Sideslip angle at vehicle CG & {$[\mathrm{rad}]$} \\
$\alpha_{v}$ & Front tire sideslip angle & {$[\mathrm{rad}]$} \\
$\alpha_{h}$ & Rear tire sideslip angle & {$[\mathrm{rad}]$} \\
$\phi$ & Roll angle measured at the roll center & {$[\mathrm{rad}]$} \\
$\dot{\phi}$ & Roll rate measured at the roll center & {$[\mathrm{rad} / \mathrm{s}]$} \\
$\dot{\psi}$ & Yaw rate at vehicle CG & {$[\mathrm{rad} / \mathrm{s}]$} \\
\hline & &
\end{tabular}

the sprung mass of the vehicle rolls about a horizontal axis along the centerline of the body.

\section{A. Single track model}

The two state linear single track model given here represents the horizontal dynamics of a car. It is also called as "the linear bicycle model" in literature and is commonly used in automotive applications. The model assumes constant velocity and small steering angle for linearization. See Fig. 1 for the representation and notation of the model, where the left hand side sketch corresponds to the lateral motion. Notice that in this model we lump left and right tires into a single one at the axle centerline hence the name "Bicycle Model" or "Single Track Model". We represent the horizontal dynamics in terms of the state variables $\beta$ and $\dot{\psi}$. The lateral tire forces $S_{v}, S_{h}$ for front and rear tires respectively, are represented as linear functions of the tire slip angles such that $S_{v}=C_{v} \alpha_{v}$, and $S_{h}=C_{h} \alpha_{h}$. Making use of Newton's 2nd law of motion one can get the following state space representation of the horizontal dynamics of the vehicle

$$
\left[\begin{array}{c}
\dot{\beta} \\
\ddot{\psi}
\end{array}\right]=\left[\begin{array}{cc}
-\frac{\sigma}{m v_{x}} & \frac{\rho}{m v_{x}^{2}}-1 \\
\frac{\rho}{J_{z z}} & -\frac{\kappa}{J_{z z} v_{x}}
\end{array}\right] \cdot\left[\begin{array}{l}
\beta \\
\dot{\psi}
\end{array}\right]+\left[\begin{array}{c}
\frac{C_{v}}{m v_{x}} \\
\frac{C_{v} l_{v}}{J_{z z}}
\end{array}\right] \delta,
$$

where the auxiliary parameters $\sigma, \rho$, and $\kappa$ are defined as follows

$$
\begin{aligned}
& \sigma \triangleq C_{v}+C_{h} \\
& \rho \triangleq C_{h} l_{h}-C_{v} l_{v} \\
& \kappa \triangleq C_{v} l_{v}^{2}+C_{h} l_{h}^{2} .
\end{aligned}
$$

We make use of this model mainly for the estimation of the uncertain model parameters $C_{v}, C_{h}, l_{v}, l_{h}$ based on the multiple model structure. Note that although (1) is linear in the state variables, it is nonlinear with respect to unknown parameter variations. 


\section{B. Roll plane model}

For the realtime estimation of $\mathrm{CG}$ height $h$ as well as the parameters of the suspension system $k$ and $c$ based on the multiple model switching method, we use the 2-state roll plane model described here. This is the simplest model that captures the roll dynamics of the car and it is free from the effects of uncertainties originating from unknown tire stiffness parameters, which in turn makes it suitable for the estimation task.

Assuming that all vehicle mass is sprung, and linear forces exerted by the suspension system are such that $F_{\text {spring }}=k \phi$, and $F_{\text {damper }}=c \dot{\phi}$, then we can apply a torque balance in the roll plane of the vehicle in terms of the suspension forces (see Fig. 1 right hand side for the schematic and the notation of the roll plane model), and obtain the following relationship

$$
J_{x_{e q}} \ddot{\phi}+c \dot{\phi}+k \phi=m h\left(a_{y} \cos \phi+g \sin \phi\right),
$$

where $J_{x_{e q}}$ is the equivalent roll moment of inertia derived using the parallel axis theorem of mechanics taking into account the CG height as described below

$$
J_{x_{e q}} \triangleq J_{x x}+m h^{2} .
$$

For small $\phi$ we can approximate nonlinear terms as $\cos \phi \approx 1$, $\sin \phi \approx \phi$ and represent this equation as in the following state space form

$$
\left[\begin{array}{l}
\dot{\phi} \\
\ddot{\phi}
\end{array}\right]=\left[\begin{array}{cc}
0 & 1 \\
-\frac{k-m g h}{J_{x_{e q}}} & -\frac{c}{J_{x_{e q}}}
\end{array}\right] \cdot\left[\begin{array}{c}
\phi \\
\dot{\phi}
\end{array}\right]+\left[\begin{array}{c}
0 \\
\frac{m h}{J_{x_{e q}}}
\end{array}\right] a_{y} .
$$

Note that at steady state one can calculate the CG height by a single model using the relationship

$$
h=\frac{k \phi}{m\left(g \phi+a_{y}\right)},
$$

given that the roll angle $\phi$, and the lateral acceleration $a_{y}$ measurements as well as an accurate knowledge of the spring stiffness $k$ are available. While former can be measured using suitable sensors, $k$ is unknown and needs to be calculated depending on the specific maneuver and loading condition, and is effected by various other factors. As will be explained in section III, using the multiple model switching method we neither need the exact knowledge of the suspension parameters, nor steady state type excitation to get an accurate estimation of the CG height. As a final remark note that although (5) is linear in the state variables, it is nonlinear with respect to unknown parameter variations of $k, c$ and $h$.

\section{Single track model with roll degree of freedom}

While we utilize the previous two models for the estimation task of the unknown vehicle parameters, we employ the linear bicycle model with roll degree of freedom described here, to generate the reference vehicle behavior. The model is the simplest model with coupled lateral and roll dynamics, which assumes that $\delta, \phi, \beta$ are small and all vehicle mass is sprung.
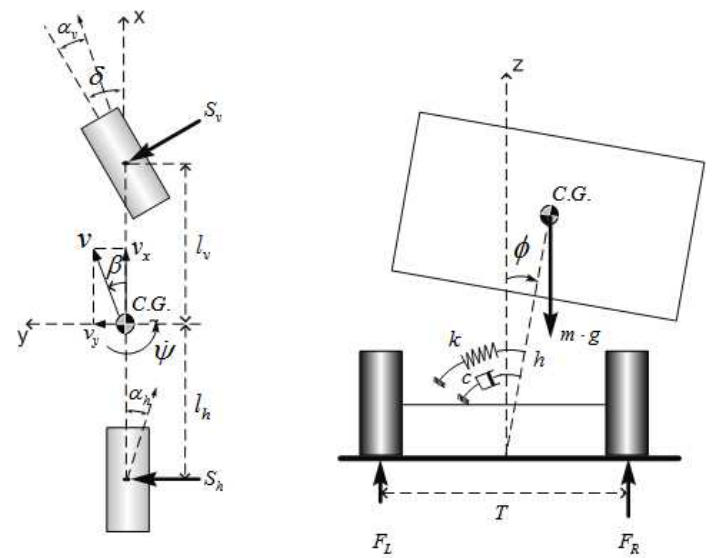

Fig. 1. Linear bicycle model with roll degree of freedom.

We can write the equations of motion for the single track model with the extended roll degree of freedom as follows

$$
\begin{array}{r}
\dot{x}=\left[\begin{array}{cccc}
-\frac{\sigma}{m v_{x}} \frac{J_{x e q}}{J_{x x}} & \frac{\rho}{m v_{x}^{2}} \frac{J_{x_{e q}}}{J_{x x}}-1 & -\frac{h c}{J_{x x} v_{x}} & \frac{h(m g h-k)}{J_{x x} v_{x}} \\
\frac{\rho}{J_{z z}} & -\frac{\kappa}{J_{z z} v_{x}} & 0 & 0 \\
-\frac{h \sigma}{J_{x x}} & \frac{h \rho}{v_{x} J_{x x}} & -\frac{c}{J_{x x}} & \frac{m g h-k}{J_{x x}} \\
0 & 0 & 1 & 0
\end{array}\right] x \\
+\left[\begin{array}{c}
\frac{C_{v}}{m v_{x}} \frac{J_{x e q}}{J_{x x}} \\
\frac{C_{v} l_{v}}{J_{z z}} \\
\frac{h C_{v}}{J_{x x}} \\
0
\end{array}\right] \delta,
\end{array}
$$

where $x=\left[\begin{array}{llll}\beta & \dot{\psi} & \dot{\phi} & \phi\end{array}\right]^{T}$ is the state vector. State responses to a step steering input are shown in Fig. 2 below, where the steering magnitude was $30^{\circ}$ with a steering ratio of $1: 18$.

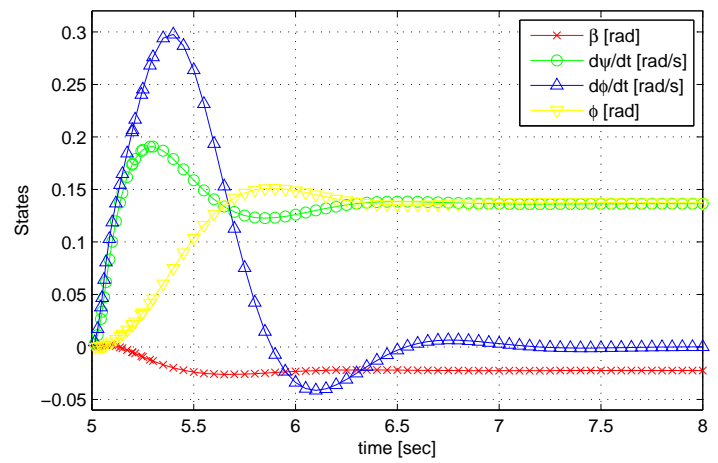

Fig. 2. State responses of the single track model with roll degree of freedom to a step steering input of $30^{\circ} / 18$ in magnitude.

\section{MUlTiPle MODELS \& SWITCHING METHOD FOR VEHICLE PARAMETER IDENTIFICATION}

Well-established linear least squares type identification techniques are the conventional approaches to parameter estimation, yet such methods are susceptible to loss of identifiability due to feedback [16], [17] as is the case for the 
problem described here. Also, the linear models introduced in preceding section are nonlinear in the unknown vehicle parameters further complicating the formulation of the estimation problem using the traditional approaches. Although linear regression techniques typically converge quickly, they tend do so more effectively when the measurement signals are persistently exciting [16], [18]. For CG estimation problem this would mean to impose some specific maneuver requirements on the driver input such that all the modes of excitation are covered and a dependable estimation of the unknown parameters could be made. Such a demand on the driver input would not only be unrealistic but also unreliable. Thus there is a need for a different approach for the parameter identification task, which imposes no restriction on the driver input, has fast convergence rates and requires minimum additional output information (sensors). Here we introduce a multiple model switching algorithm [5] to identify unknown vehicle parameters rapidly in real-time, which meets the estimation requirements described earlier. In what follows, we take a modular approach of decoupling the vehicle dynamics into subsystems by assuming a weak relationship between the lateral and roll dynamics. We then present our methodology and give numerical simulation results corresponding to the decoupled identification algorithms.

\section{A. Realtime estimation of longitudinal CG position}

In order to estimate the longitudinal CG position $l_{v}$ along with the tire stiffness parameters $C_{v}$ and $C_{h}$, here we introduce the multiple model \& switching estimation algorithm, which makes use of the lateral dynamics model (1). We assume that each unknown model parameter belongs to a closed interval such that $C_{v} \in \mathscr{C}_{v}, C_{h} \in \mathscr{C}_{h}$, and $l_{v} \in \mathscr{L}_{v}$. These intervals are divided into certain number of grid points and they can be represented as $\mathscr{C}_{v}=\left\{C_{v_{1}}, C_{v_{2}}, C_{v_{3}}, \ldots, C_{v_{p}}\right\}, \mathscr{C}_{h}=$ $\left\{C_{h_{1}}, C_{h_{2}}, C_{h_{3}}, \ldots, C_{h_{q}}\right\}$, and $\mathscr{L}_{v}=\left\{l_{v_{1}}, l_{v_{2}}, l_{v_{3}}, \ldots, l_{v_{r}}\right\}$ with dimensions $p, q$ and $r$ respectively.

As the basis of the multiple model scheme, we form $n=p \times$ $q \times r$ different models corresponding to the cross combinations of the grid points in the parameter space. Utilizing (1) the equations of motion corresponding to each model can be represented as

$$
\left[\begin{array}{c}
\dot{\beta}_{i} \\
\ddot{\psi}_{i}
\end{array}\right]=\left[\begin{array}{cc}
-\frac{\sigma_{i}}{m v_{x}} & \frac{\rho_{i}}{m v_{x}^{2}}-1 \\
\frac{\rho_{i}}{J_{z z}} & -\frac{\kappa_{i}}{J_{z z} v_{x}}
\end{array}\right] \cdot\left[\begin{array}{c}
\beta_{i} \\
\dot{\psi}_{i}
\end{array}\right]+\left[\begin{array}{c}
\frac{\left(C_{v}\right)_{i}}{m v_{x}} \\
\frac{\left(C_{v}\right) i v_{i}}{J_{z z}}
\end{array}\right] \delta,
$$

where $i=1,2, \ldots, n$ denotes the model number. We assume that all models have zero initial conditions such that $\beta_{i}(0)=0$, and $\dot{\psi}_{i}(0)=0$, for $i=1,2, \ldots, n$. Furthermore, each model is driven by the same inputs $\delta$ and $v_{x}$ as depicted in Fig. 3 , measurements of which are assumed to be provided by suitable set of sensors. In order to select the model with the correct parametrization we utilize the identification error, which is the difference between the model and the plant outputs. Mathematically, we can express the identification error $e_{i}$ corresponding $i^{t h}$ model as

$$
e_{i}=y_{\text {plant }}-\left(y_{\text {model }}\right)_{i},
$$

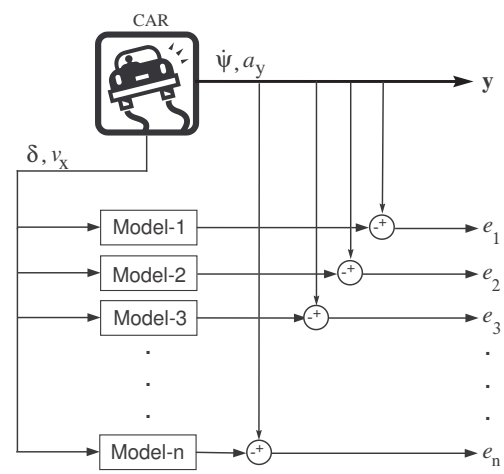

Fig. 3. Multiple Model System Identification Algorithm.

where $y$ denotes the model or the plant output. For the longitudinal CG position estimation problem, we select the model and plant outputs as $y=\left[a_{y}, \dot{\psi}\right]$, measurements of which are available through a suitable set of sensors. Thus we can represent the identification error for the $i^{\text {th }}$ model as follows

$$
e_{i}(t)=\left[\begin{array}{c}
a_{y}(t)-a_{y, i}(t) \\
\dot{\psi}(t)-\dot{\psi}_{i}(t)
\end{array}\right], \quad i=1,2, \ldots, n .
$$

Inspired by the quadratic cost optimization techniques, we next compute a cost function $J_{i}$ corresponding to the $i^{\text {th }}$ identification error as in the following form

$$
J_{i}(t)=\alpha\left\|e_{i}(t)\right\|+\beta \int_{0}^{t} e^{-\lambda(t-\tau)}\left\|e_{i}(\tau)\right\| d \tau,
$$

which was originally suggested by Narendra et al. in [4] as a switching scheme. In this cost function $\alpha$, and $\beta$ are free design parameters controlling the relative weights given to transient and steady state measures respectively, whereas $\lambda$ is the forgetting factor. Note that it is possible to use other type of cost functions depending on the specific estimation requirements from the problem at hand. Here we selected the model with the least cumulative identification error according to (11) using

$$
i^{\star}=\arg \min _{i=1, \ldots, N} J_{i}(t) .
$$

Within the parameter space described by $\mathscr{C}_{v}, \mathscr{C}_{h}$ and $\mathscr{L}_{v}$, selected model $i^{\star}$ and the corresponding model parameters $C_{v}^{\star}, C_{h}^{\star}$ and $l_{v}^{\star}$ have the minimum cumulative distance to the parameters of the plant.

Numerical Analysis: In the following figures we present the estimation results for the algorithm based on simulated sensor signals generated by the vehicle model (7). The reference model parameters used are given in Table II.

The maneuver was conducted at $108 \mathrm{~km} / \mathrm{h}$, and as seen in Fig. 4 for the steering input plot, the maneuver tested was an obstacle avoidance maneuver commonly known as the elktest, with a peak magnitude of $30^{\circ}$ at the steering wheel (the steering ratio is $1 / 18$ between the tires and the steering wheel). The model space consisted of 140 models in total. The uniformly distributed parameter space were selected as $\mathscr{C}_{v}=$ 
TABLE II

REFERENCE MODEL PARAMETERS

\begin{tabular}{cll}
\hline parameter & value & unit \\
\hline$m$ & 1300 & {$[\mathrm{~kg}]$} \\
$g$ & 9.81 & {$\left[\mathrm{~m} / \mathrm{s}^{2}\right]$} \\
$v_{x}$ & 30 & {$[\mathrm{~m} / \mathrm{s}]$} \\
$\delta_{\text {peak }}$ & $30 \cdot \frac{1}{18}$ & {$[\mathrm{deg}]$} \\
$J_{x x}$ & 400 & {$\left[\mathrm{~kg} \cdot \mathrm{m}^{2}\right]$} \\
$J_{z z}$ & 1200 & {$\left[\mathrm{~kg} \cdot \mathrm{m}^{2}\right]$} \\
$l_{v}$ & $\mathbf{1 . 2}$ & {$[\mathrm{m}]$} \\
$l_{h}$ & 1.3 & {$[\mathrm{~m}]$} \\
$L$ & 2.5 & {$[\mathrm{~m}]$} \\
$h$ & $\mathbf{0 . 7}$ & {$[\mathrm{m}]$} \\
$c$ & 5000 & {$\left[\mathrm{~kg} \cdot \mathrm{m}^{2} / \mathrm{s}\right]$} \\
$k$ & 36000 & {$\left[\mathrm{~kg} \cdot \mathrm{m}^{2} / \mathrm{s}^{2}\right]$} \\
$C_{v}$ & 60000 & {$[\mathrm{~N} / \mathrm{rad}]$} \\
$C_{h}$ & 90000 & {$[\mathrm{~N} / \mathrm{rad}]$} \\
\hline & &
\end{tabular}

[50000, 80000] with intervals of $10000, \mathscr{C}_{h}=[60000,100000]$ with intervals of 10000 corresponding to the range of tire stiffness parameters, and $\mathscr{L}_{v}=[1,1.6]$ with intervals of 0.1 corresponding to the space of possible longitudinal CG positions.

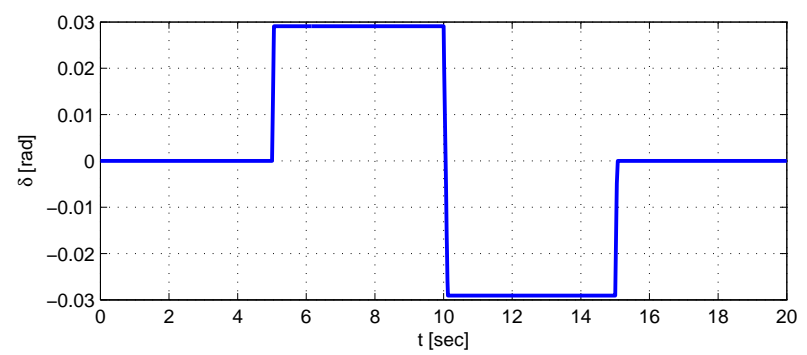

Fig. 4. Steering input

In Fig. 5 the simulated sensor data is compared against the selected model outputs, while in Fig. 6 the corresponding longitudinal CG position estimation is presented. The discontinuous jumps in Fig. 6 occur due to the switching between the models. We observe that based on the simulated measurement data, the multiple model switching algorithm successfully estimated the longitudinal CG location to be $1.2 \mathrm{~m}$, precisely matching the reference model. When there is no exact model match in the parameter space, a small offset is expected due to the unique shape of the selected cost function (11) in the parameter space. However, with proper selection and/or redistribution of models, one can overcome this problem to yield the closest model match.

\section{B. Realtime estimation of $C G$ height}

Here we present the multiple model switching algorithm to estimate CG height $h$ along with the linear suspension parameters $k$ and $c$ based on the roll-plane model (5). We assume that each unknown parameter belongs to a closed interval such that $h \in \mathscr{H}, k \in \mathscr{K}$, and $c \in \mathscr{C}$. These intervals are divided into sufficient number of grid points and they can be represented as $\mathscr{H}=\left\{h_{1}, h_{2}, h_{3}, \ldots, h_{p}\right\}, \mathscr{K}=\left\{k_{1}, k_{2}, k_{3}, \ldots, k_{q}\right\}$, and $\mathscr{C}=$
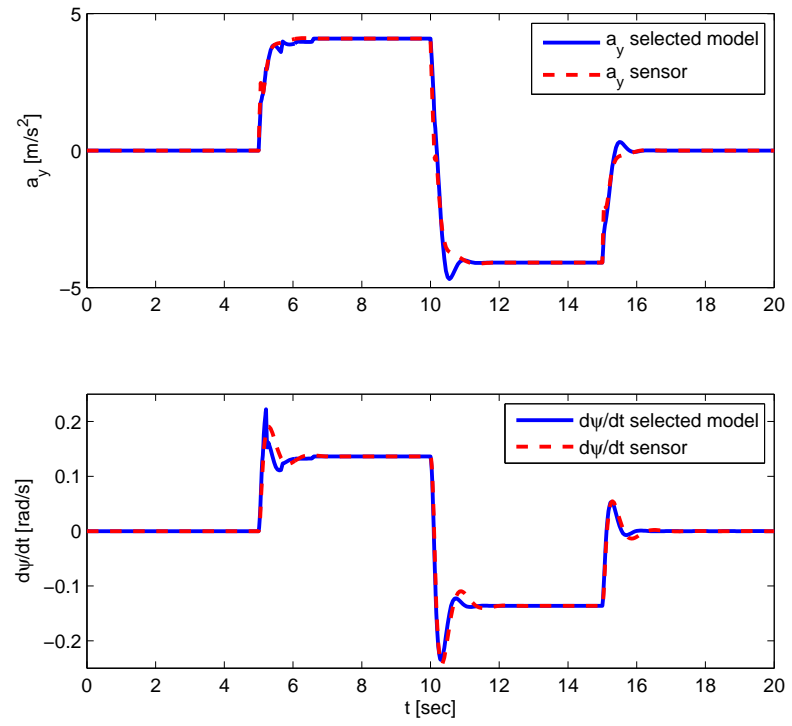

Fig. 5. Sensor and the selected model output comparison for the longitudinal CG position estimation

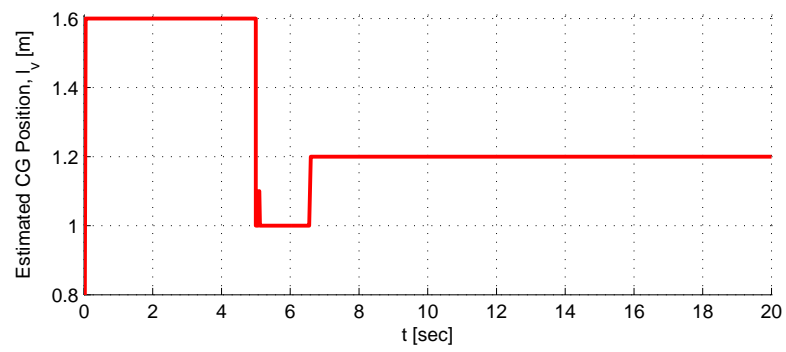

Fig. 6. Longitudinal $\mathrm{CG}$ position estimation with exact match

$\left\{c_{1}, c_{2}, c_{3}, \ldots, c_{r}\right\}$ with dimensions $p, q$ and $r$ respectively. We then form $n=p \times q \times r$ different models corresponding to the cross combinations of the grid points in the parameter space. Utilizing (5) the equations of motion corresponding to each model can be represented as

$$
\left[\begin{array}{c}
\dot{\phi}_{i} \\
\ddot{\phi}_{i}
\end{array}\right]=\left[\begin{array}{cc}
0 & 1 \\
-\frac{k_{i}-m g h_{i}}{J_{x e q}, i} & -\frac{c_{i}}{J_{x e q}, i}
\end{array}\right] \cdot\left[\begin{array}{c}
\phi_{i} \\
\dot{\phi}_{i}
\end{array}\right]+\left[\begin{array}{c}
0 \\
\frac{m h_{i}}{J_{x e q}, i}
\end{array}\right] a_{y}
$$

where $i=1,2, \ldots, n$ denotes the model number. We assume that all models have zero initial conditions such that $\phi_{i}(0)=0$, and $\dot{\phi}_{i}(0)=0$, for $i=1,2, \ldots, n$. Similar to what is shown in Fig. 3, every model is driven by the same input $a_{y}$, which is measured. According to (9) we again calculate identification errors $e_{i}$, however this time the plant and model outputs to compare are the roll angles, as follows

$$
e_{i}(t)=\phi(t)-\phi_{i}(t), \quad i=1,2, \ldots, n .
$$

Note that one can also include the roll rate $\dot{\phi}$ measurement if available, in the output vector. However, the influence of $\dot{\phi}$ on the estimation results were relatively insignificant as compared 
to the roll angle $\phi$, thus was omitted here. This is also in accordance with our assumption of no additional sensors to the available ones.

Now one can compute cost functions (11) corresponding to each identification error. Switching among the models based on (12) yields the one with the minimum cumulative identification error and the selected $k^{\star}, c^{\star}$ and $h^{\star}$ represent the plant in the parameter space described by $\mathscr{K}, \mathscr{C}$ and $\mathscr{H}$ respectively.

Numerical Analysis: The CG height estimation results for the simulated measurement data described in the previous subsection is given here. The model space consisted of 240 models in total, where the uniformly distributed parameter space were selected as $\mathscr{K}=[30000,40000]$ with intervals of $2000, \mathscr{C}=[4000,6000]$ with intervals of 500 corresponding to the parameter space for suspension parameters, and $\mathscr{H}=$ $[0.5,0.85]$ with intervals of 0.05 corresponding to the range of possible CG heights. In Fig. 7 sensor and the switched model outputs are compared whereas in Fig. 8 the CG height estimation results are shown. Based on the results, we again observe that the multiple model switching algorithm successfully estimated the CG height to be $0.7 \mathrm{~m}$, precisely matching the reference vehicle data.

Based on the simulation results presented in the preceding and current subsections we conclude that the estimation method gives fast and accurate results, which makes it suitable for use in active automotive handling control.
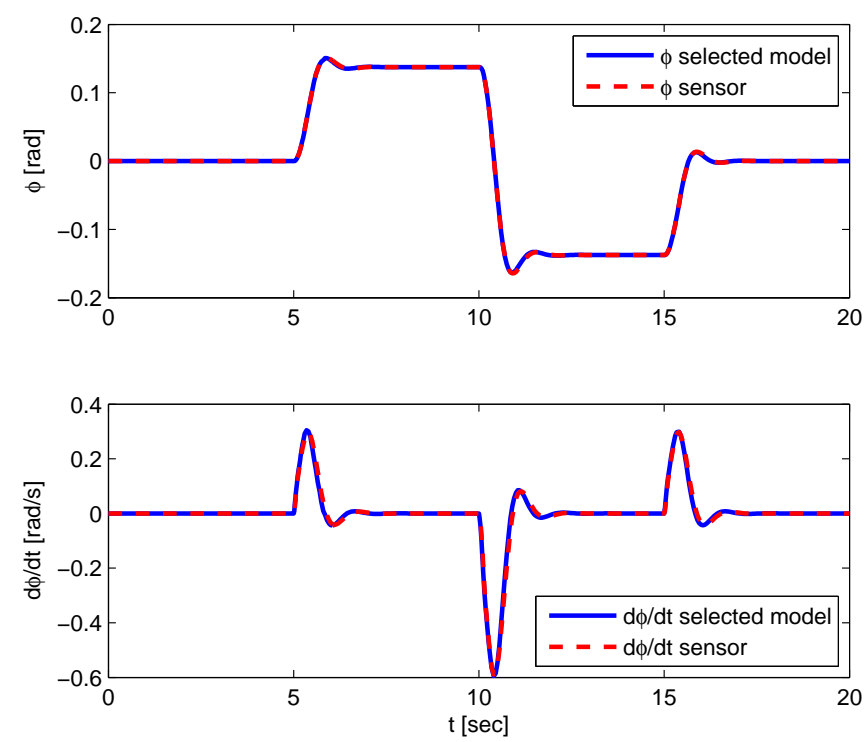

Fig. 7. Sensor and the selected model output comparison for the CG height estimation

\section{AN APPLICATION EXAMPLE: LOAD CONDITION ESTIMATOR}

In this section we introduce a problem related to rollover prevention for implementing our estimation technique. The problem originates from a particular robust rollover controller

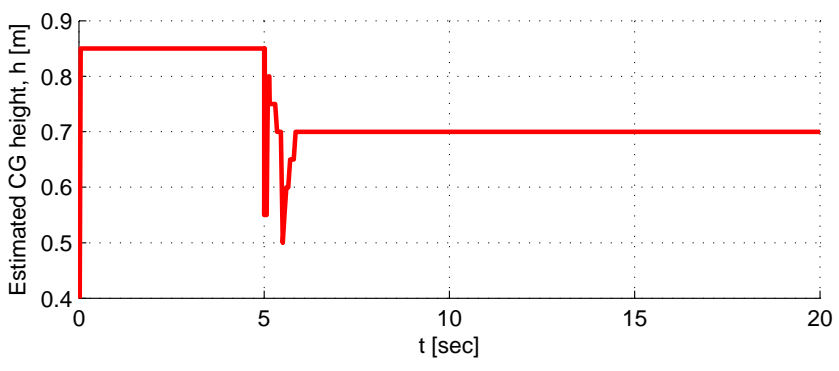

Fig. 8. CG height estimation with exact match

design in an SUV class vehicle such that when the vehicle is empty excluding the weight of driver, there is no risk of un-tripped rollover. In this case, a possible intervention of the controller results in a loss of performance and must be avoided. In what follows, we give a version of the multiple model \& switching algorithm to estimate whether the load condition of the vehicle is above the threshold weight. The threshold weight here is defined by the total weight of the empty vehicle and the driver. For this problem we employed the roll plane model (5) and further assumed the availability of the set of the roll angle $(\phi)$, and the lateral acceleration $\left(a_{y}\right)$ sensors. We also assumed that we know the parameters of the vehicle corresponding to the threshold loading condition.

For the multiple model switching algorithm we set the known mass $m$, CG height $h$, damping coefficient $c$, and roll moment of inertia $J_{x x}$ corresponding to the threshold loading condition to be the same in every model, where the models are parameterized with different spring stiffnesses. We assumed that spring stiffness belongs to a closed interval such that $k \in \mathscr{K}$, where the interval is divided into $n$ grid points such that $\mathscr{K}=\left\{k_{1}, k_{2}, k_{3}, \ldots, k_{n}\right\}$. In other words we have $n$ different models corresponding to the different $k$ values. The equations of motion for the models with zero initial conditions can be expressed with (13). While each model is driven by the same input $a_{y}$, the corresponding identification errors $e_{i}$ are calculated according to (14). Given this setup, one can compute cost functions (11) corresponding to each identification error and switching among the models based on (12) yields the one with the minimum cumulative identification error. The selected $k^{\star}$ represent the plant in the parameter space $\mathscr{K}$, and if it is different than that of the vehicle with threshold load condition then we can conclude that there is more load on the vehicle than the threshold value.

Numerical Analysis: In our simulations we chose parameters given in Table II to represent the threshold loading of the vehicle. We also used the same obstacle avoidance maneuver introduced in the preceding section, at the speed of $108 \mathrm{~km} / \mathrm{h}$ and with a steering profile as shown in Fig. 4 . We tested 9 different loading scenarios as described in Table III, where the first case corresponds to the threshold loading condition. The model space consisted of 11 models in total, where the uniformly distributed parameter space was chosen as $\mathscr{K}=[30000,40000]$ with intervals of 1000 . Based on the 
TABLE III

LOADING SCENARIOS

\begin{tabular}{|c||c|c|c|}
\hline Case & Weight $[\mathrm{kg}]$ & CG height $[\mathrm{m}]$ & Threshold Loading? \\
\hline \hline $\mathbf{1}$ & $\mathbf{1 3 0 0}$ & $\mathbf{0 . 7 0}$ & yes \\
2 & 1350 & 0.70 & no \\
3 & 1400 & 0.70 & no \\
4 & 1450 & 0.70 & no \\
5 & 1500 & 0.70 & no \\
6 & 1300 & 0.75 & no \\
7 & 1300 & 0.80 & no \\
8 & 1300 & 0.85 & no \\
9 & 1300 & 0.90 & no \\
\hline
\end{tabular}

described algorithm, only the first case was recognized as the threshold loading condition, and the recognition took less then 1.5 seconds into the maneuver in all the cases.

Based on the results, we conclude that this version of the multiple model \& switching algorithm can successfully be used to rapidly recognize a specific loading condition of the vehicle based on the dynamics of the car alone, and utilizing only a small number of models.

\section{CONCLUSIONS AND FUTURE WORK}

In this paper we presented a multiple model $\&$ switching approach to realtime parameter estimation as applied to online CG location estimation in road vehicles. Numerical simulation results demonstrate the efficacy of the suggested technique in terms of estimation speed and accuracy. Also the load condition estimator example demonstrated that a version of the suggested algorithm can easily be integrated into current rollover or lateral dynamics controllers to enhance their performance. In the follow-up of this work we shall compare our technique to the instrumental-variable type methods to fully assess its potential. Furthermore, we shall look into adaptive versions of the multiple model algorithm to deal with the case when the parameter sets do not include the exact vehicle parameterizations. Finally, with the scope of testing the algorithm in a real vehicle, we shall develop and integrate our method with direct rollover prevention and lateral dynamics controllers and compare their performance to that of alternative robust control approaches.

\section{ACKNOWLEDGEMENTS}

This work was partially supported by Science Foundation Ireland Grant 04/IN1/I478. Science Foundation Ireland is not responsible for any use of data appearing in this publication. We also would like to thank Dr. Jens Kalkkuhl and $\mathrm{Mr}$. Avshalom Suissa for their suggestions.

\section{REFERENCES}

[1] National Highway Trafic Safety Administration (NHTSA), 2006, Trafic Safety Facts 2004: A Compilation of Motor Vehicle Crash Data from the Fatality Analysis Reporting System and the General Estimates System, Technical Report.

[2] Hac A., Brown T. and Martens J., 2004, Detection of Vehicle Rollover. Vehicle Dynamics \& Simulation, SAE Technical Paper Series.

[3] Carlson C.R. and Gerdes J.C., 2003, Optimal Rollover Prevention with Steer by Wire and Differential Braking. Proceedings of IMECE, Nov. 16-21, Washington D.C. USA.
[4] Narendra K.S. and Balakrishnan J., 1994, Improving Transient Response of Adaptive Control Systems using Multiple Models and Switching. IEEE Transactions on Automatic Control, Vol. 39, No. 9.

[5] Akar M., Solmaz S. and Shorten R., 2006, Method for Determining the Center of Gravity for an Automotive Vehicle, Irish Patent.

[6] Mango N., 2004, Measurement \& Calculation of Vehicle Center of Gravity Using Portable Wheel Scales. SAE World Congress, Mar. 8-11, Detroit, MI USA.

[7] Allen R.W., Klyde D.H., Rosenthal T.J., and Smith D.M., 2003, Estimation of Passenger Vehicle Inertial Properties and Their Effect on Stability and Handling. Journal of Passenger Cars-Mechanical Systems, Vol. 112.

[8] Chen B. and Peng H., 2001, Differential-Breaking-Based Rollover Prevention for Sport Utility Vehicles with Human-in-the-loop Evaluations. Vehicle System Dynamics, Vol.36, No.4-5, pp 359-389.

[9] Wielenga T.J., 1999, A Method for Reducing On-Road Rollovers: Anti -Rollover Braking. SAE Paper No. 1999-01-0123.

[10] Palkovics L., Semsey À. and Gerum E., 1999, Roll-Over Prevention System for Commercial Vehicles-Additional Sensorless Function of the Electronic Brake System. Vehicle System Dynamics, Vol.4, pp.285-297.

[11] Kamnik R., Böttiger F., Hunt K., 2003, Roll Dynamics and Lateral Load Transfer Estimation in Articulated HeavyFreight Vehicles: A Simulation Study. Proceedings of the Institution of Mechanical Engineers, Part D.

[12] Ackermann J. and Odenthal D., 1998, Robust steering control for active rollover avoidance of vehicles with elevated center of gravity. Proc. of International Conference on Advances in Vehicle Control and Safety, (Amiens, France).

[13] Odenthal D., Bünte T. and Ackermann J., 1999, Nonlinear steering and breaking control for vehicle rollover avoidance. European Control Conference, (Karlsruhe, Germany).

[14] Kiencke U. and Nielsen L., 2000, Automotive Control Systems for Engine, Driveline and Vehicle, (Springer-Verlag \& SAE Int., Berlin).

[15] Schindler E., 2001, Vehicle Dynamics, Lecture notes, FHTE Esslingen University of Applied Sciences, Esslingen.

[16] Söderström T., Stoica P., 1989, System Identification, Prentice Hall International, International Series in Systems and Control Engineering, (Cambridge, Great Britain).

[17] Àström K.J., Wittenmark B., 1995 Adaptive Control, 2nd edition, (Addison-Wesley Publ Co).

[18] Narendra K. S., Annaswamy A. M., 1989, Stable Adaptive Systems, (Prentice Hall International, Englewood Cliffs - NJ, U.S.A.). 